\title{
Recomendações do uso da ventilação mecânica para crianças em suspeita ou confirmação de COVID-19*
}

\author{
Recommendations for the use of mechanical ventilation in children with suspected or confirmed \\ COVID-19*
}

RIBEIRO, Simone Nascimento Santos ${ }^{1}$; ANDRADE, Lívia Barboza de ${ }^{2}$; SOUSA, Mayson Láercio de Araújo ${ }^{3}$; SOUZA, Guilherme Cherene Barros de ${ }^{4}$; LANZA, Fernanda de Cordoba 5 .

\begin{abstract}
Resumo
Este documento tem por objetivo abordar os principais dados de suporte ventilatório, da ventilação mecânica em pacientes pediátricos com COVID-19. As manifestações clínicas da doença grave de COVID-19 em crianças ainda não foram completamente elucidadas, mas não se pode descartar que uma proporção de pacientes possa apresentar características clínicas muito parecidas com a bronquiolite, broncopneumonia, asma, pneumonite que são observadas em outras doenças respiratórias virais da infância. As informações dos casos que necessitam de cuidados intensivos e uso de ventilação mecânica são muito escassos e limitados, de maneira geral, os dados são compatíveis com uma infecção viral respiratória semelhante a outras. O consenso de especialistas Paediatric Mechanical Ventilation Consensus Conference (PEMVECC) e Pediatric Acute Lung Injury Consensus Conference (PALICC), apontam a adesão às recomendações sobre ventilação mecânica em crianças publicadas anteriormente e às recomendações práticas nas questões específicas relacionadas à doença de COVID-19 em crianças.
\end{abstract}

Palavras-chave: Fisioterapia; ventilação mecânica pediátrica; ventilação não invasiva; COVID-19.

* Revisado por membros do Comitê COVID-19 da ASSOBRAFIR, nomeado por meio do memorando Nº03/2020. Esta publicação é uma atualização da Comunicação Oficial "Recomendações do uso da Ventilação Mecânica para crianças em suspeita ou confirmação de COVID-19", chancelada pelo Comitê COVID-19 da ASSOBRAFIR, originalmente escrita pelos mesmos autores e divulgada em 08/05/2020 no endereço eletrônico https://assobrafir.com.br/wp-content/ uploads/2020/05/ASSOBRAFIR COVID-19 VM-PED 2020.05.07.pdf

1 Instituto de Previdência dos Servidores do Estado de Minas Gerais - IPSEMG. Departamento de Fisioterapia da Faculdade Ciências Médicas de Minas Gerais - CMMG. Belo Horizonte - MG, Brasil. Email: simonensribeiro@gmail.com. SNSR https://orcid.org/0000-0002-9364-7836

2 Programa de pós-graduação, Instituto de Medicina Integral Prof. Fernando Figueira. Recife - PE, Brasil. LBA - https://orcid. org/0000-0002-5647-1835

3 Serviço de Fisioterapia, Instituto do Coração, Hospital das Clínicas HCFMUSP, Faculdade de Medicina Universidade de São Paulo - SP, Brasil. MLAS - http://orcid.org/0000-0003-4236-5107

${ }^{4}$ Serviço de Fisioterapia Hospital Norte D Or. Rio de Janeiro - RJ, Brasil. GCNS - https://orcid.org/0000-0003-1869-1172

5 Programa de Pós-Graduação em Ciências da Reabilitação e Departamento de Fisioterapia da Universidade Federal de Minas Gerais -UFMG. Belo Horizonte - MG, Brasil. FCL - https://orcid.org/0000-0002-4748-6947 


\section{Abstract}

This document aims to address the available data on ventilatory support and mechanical ventilation in pediatric patients with COVID-19. The clinical manifestations of severe COVID-19 disease in children have not yet been fully elucidated, but it cannot be ruled out that a proportion of patients may present with clinical characteristics very similar to bronchiolitis, bronchopneumonia, asthma or pneumonitis, conditions which are frequently observed in other childhood respiratory virus diseases. Information on cases requiring intensive care and about the use of mechanical ventilation is very scarce and limited. In general, these pieces of information seem to be similar to those used in other respiratory virus infections. The expert consensus documents from the Pediatric Mechanical Ventilation Consensus Conference (PEMVECC) and Pediatric Acute Lung Injury Consensus Conference (PALICC) recommend to follow previously published recommendations on mechanical ventilation in children and practical recommendations on specific issues related to COVID-19 in children.

Keywords: Physiotherapy; Mechanical Ventilation; Non invasive ventilation; Pediatric; COVID-19.

\section{Objetivo}

O objetivo do presente posicionamento é fornecer direcionamentos para o atendimento de Fisioterapia a pacientes pediátricos com COVID-19, com foco no suporte ventilatório durante a intervenção que dependerá a gravidade da doença.

\section{Recomendações do Uso Ventilação Mecânica para Crianças em Suspeita ou Confirmação de COVID-19}

Desde fevereiro de 2020 o Brasil foi incluído nos números da pandemia mundial da doença COVID-19, ${ }^{1}$ que teve início na China em dezembro de 2019. ${ }^{2}$ Não há números detalhados sobre o acometimento na população infantil no Brasil, mas dados mundiais apresentam que menor número de crianças e adolescentes terão insuficiência respiratória por COVID $-19^{3}$ e necessitarão de tratamento em terapia intensiva, comparado aos adultos. Para os pacientes mais graves haverá necessidade de instalar ventilação mecânica invasiva (VM) para garantir a troca gasosa. Diante dessas considerações, esse documento tem por objetivo recomendar estratégias ventilatórias para uso de VM em crianças com suspeita, ou confirmação de COVID-19.

\section{Epidemiologia}

Estima-se que cerca de 1\% do total de acometidos pelo vírus SARS-Cov2 seja de crianças abaixo de 10 anos. ${ }^{3}$ A sintomatologia da doença COVID-19 tem sido mais branda na população infantil. ${ }^{4}$ Há diversas hipóteses para a menor gravidade, dentre elas a menor expressão do receptor da enzima conversora de angiotensina 2 (ECA2) de crianças. A ECA2, receptor necessário para combater a infecção pode SARS-Cov2, não está presente nas células de defesa das crianças, o que resulta em quadro clínico diferenciado, quando comparado aos adultos. ${ }^{4,5}$

A transmissão na população infantil se dá pelo contato com mucosas e trato respiratório, ao falar, tossir e espirrar. ${ }^{5}$ A transmissão vertical mãe-filho ou por amamentação ainda não está bem estabelecida.

Dados da China apontam que menos de 5\% do total pacientes com COVID-19 eram crianças. ${ }^{6}$ A sintomatologia varia de infecção leve das vias aéreas superiores até sintomas mais graves, com possibilidade haver insuficiência respiratória e síndrome do desconforto respiratório. Inicialmente, 
a tosse, febre e falta de ar estão presentes, e diarreia e vômitos podem ser observados nas crianças. A progressão dos sintomas de COVID-19 é rápida, entre 1 a 3 dias, em especial nos pacientes que apresentam fatores de risco, como doenças crônicas associadas. Cabe ressaltar, que quase $30 \%$ dos casos serão assintomáticos, e apenas identificados pois, algum indivíduo da família apresentou a doença. ${ }^{6}$

Na população infantil a incubação do vírus varia entre 5 a 10 dias, e a duração dos sintomas é acima de 10 dias na maior parte dos pacientes, sendo maior que 20 dias para aqueles com doença crônica associada. ${ }^{5} \mathrm{~A}$ recuperação se dá entre 5 dias a 2 semanas. ${ }^{6}$

A sazonalidade das doenças respiratórias virais é importante aspecto a observar. Nesse momento, no Brasil, estamos no outono, período que há aumento no número de casos de bronquiolite nos lactentes por Vírus Sincicial Respiratório, Influenza, entre outros. Considerando a similaridade da sintomatologia entre as doenças, o histórico deve ser levado em consideração para que o diagnóstico seja assertivo. A maior parte dos pacientes pediátricos infectados estão em famílias que apresentam, ao menos, um adulto com sintomas da COVID-19. ${ }^{4}$

\section{Classificação da síndrome do desconforto respiratório agudo em pediatria (SDRAp)}

Pacientes pediátricos que pioram gradativamente o quadro clínico, apresentam aumento na frequência respiratória (maior que 60rpm para lactentes abaixo de 2 meses de vida; maior que 50rpm entre 2 e 12 meses; maior que 40rpm entre 1 e 5 anos; maior que 30 para acima de 5 anos) $)^{5}$ devem ser acompanhados em ambiente hospitalar.

A Sociedade Europeia de Terapia Intensiva Pediátrica e Neonatal apresentou um painel de sugestões para abordar crianças que cursam com insuficiência respiratória por COVID-19, visto que estas apresentam um quadro semelhante à Síndrome do Desconforto Respiratório Agudo - SDRA ${ }^{7}$. Assim as recomendações de intervenções devem ser baseadas nos documentos publicados do Paediatric Mechanical Ventilation Consensus Conference (PEMVECC) e Pediatric Acute Lung Injury Consensus Conference (PALICC). Para identificar a gravidade do paciente que está em respiração espontânea, deve-se utilizar o índice de saturação de oxigênio (ISO), ou o índice de oxigenação (IO) para aqueles que estão em VM.

\section{Característica de pacientes em risco de SDRAp}

\begin{tabular}{|c|}
\hline Achados torácicos de \\
novos infiltrados \\
consistentes com \\
doença \\
parenquimatosa \\
pulmonar aguda \\
\hline
\end{tabular}
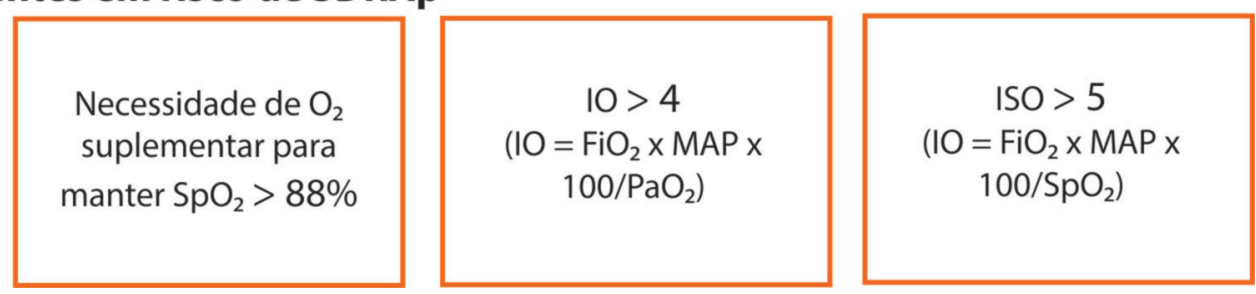

Para tratamento inicial, a pressão positiva contínua nas vias aéreas é sugerida (CPAP) ou VNI, em detrimento da cânula nasal de alto fluxo (CNAF), pois essas estratégias geram menor dispersão de aerossóis, evitando a contaminação da equipe. ${ }^{7}$ Adicionalmente, no CPAP e na VNI, maiores valores de pressão são possíveis, além do melhor controle dessa variável.

$\mathrm{Na}$ vigência de piora do quadro clínico, a intubação ortotraqueal NÃO deve ser protelada. Quando a relação $\underline{\mathrm{SpO}}_{2} / \underline{\mathbf{F i O}}_{2} \leq \mathbf{2 2 1}$, a IOT deve ser requerida. ${ }^{7}$ 
$\mathrm{A} \mathrm{SpO}_{2}$ alvo deve ser entre 92 - 97\% para pacientes em respiração espontânea, e acima de $88 \%$ para pacientes com SDRAP grave

\section{Transição da VNI para VM}

Já descrito anteriormente em documentos oficiais da ASSOBRAFIR ${ }^{8}$, devemos avaliar cada caso clínico individualmente, a fim de se definir se a VNI será o melhor recurso a ser estabelecido, em detrimento ao expressivo aumento da aerolização, quando realizado com máscara ventilada (com válvula exalatória acoplada) e consequente exposição a carga viral da equipe multidisciplinar e demais pacientes internados.

Está indicado início na VNI, o paciente que apresentar $\mathrm{SpO}_{2}<94 \%$ e $\mathrm{PaO}_{2} 63-75 \mathrm{mmHg}$ e desconforto respiratório. Está contra indicada as situações a seguir ${ }^{9,10}$ :

\section{Contraindicações da VNI}

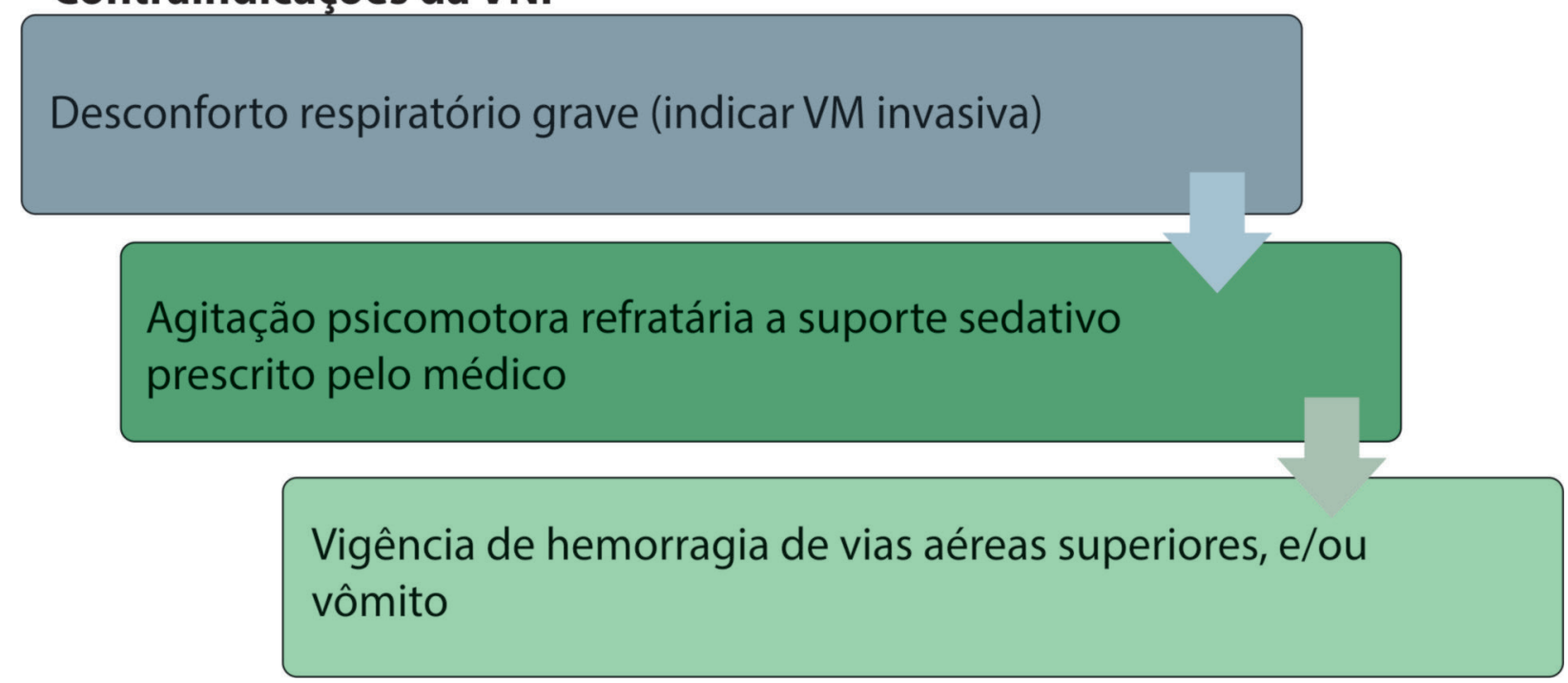

Está bem relatado a alta taxa de transmissibilidade do vírus SARS-Cov $2^{11}$, portanto, recomenda se estrutura predial adequada para realização, com segurança, da VNI, com leito de pressão negativa e filtro bacteriológico High Efficiency Particulate Air (HEPA) no ramo expiratório8.

A VNI deverá ser realizada em ventilador mecânico microprocessado que possua módulo ventilatório de dois níveis pressóricos (Binível), em circuito de ramo duplo, preferencialmente com utilização de máscara total face não ventilada (sem válvula exalatória), com filtro HME posicionado entre a máscara e o conector em $\mathrm{Y}$ e filtro bacteriológico Heat and moisture exchanger (HEPA) entre o final do ramo expiratório e a válvula exalatória do ventilador mecânico.

A estratégia ventilatória de admissão na VNI, deverá ter caráter gentil, com período de avaliação inicial em 15/ 30 (em média) minutos e reavaliações programadas em até 120 minutos (em média). Caso apresente falha dentro do período máximo, recomenda se a intubação orotraqueal. 
A estratégia ventilatória deverá respeitar os seguintes critérios:

- Avaliar se a VNI será em caráter contínuo ou intermitente, de acordo com:

o Colaboração ou agitação psicomotora;

o Desconforto respiratório antes e após adaptação da VNI

- Iniciar ventilação em modo assistido controlado $(\mathrm{S} / \mathrm{T})$ com suporte inicial de frequência respiratória em $50 \%$ da prevista para idade (avaliando o ajuste fino quando possível de tempo inspiratório, relação i:e e Rise Time);

- Volume corrente alvo em $6 \mathrm{ml} / \mathrm{kg}$;

- Menor $\mathrm{FiO}_{2}$ e PEEP possíveis para que $\mathrm{SpO}_{2} \geq 94 \%$

O sucesso da estratégia ventilatória de admissão, será determinado através de reavaliações em 15 , 30, 60, 90 e 120 minutos (em média) com monitorização dos seguintes sinais:

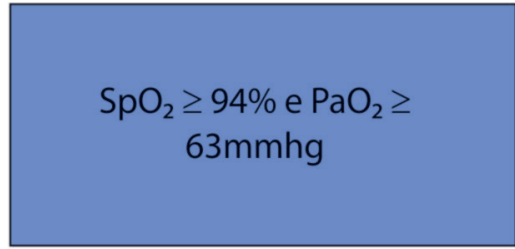

Frequência respiratória menor do que a espontânea antes da adaptação da VNI
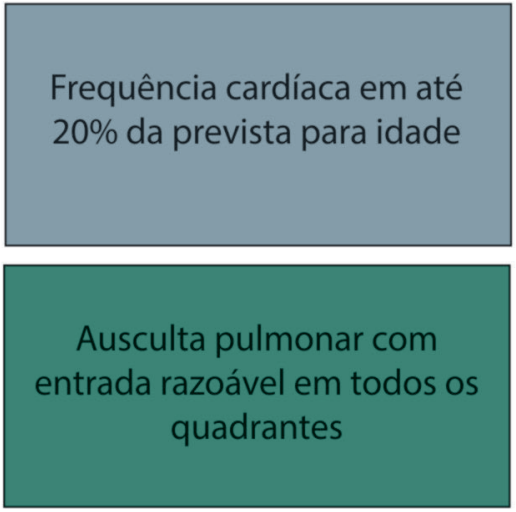

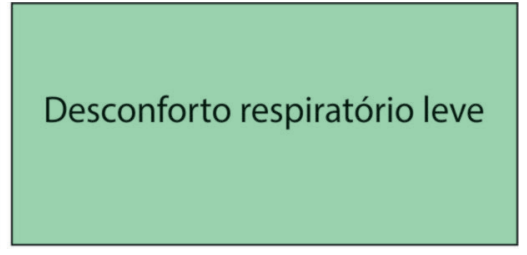

Nível de consciência e agitação psicomotora adequados para realização da VNI

A falha da VNI deverá ser considerada, caso paciente apresente os seguintes sinais de deterioração clínica (em até 120 minutos iniciais - em média), podendo considerar possível intubação orotraqueal:

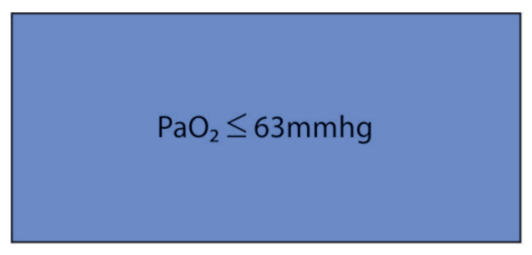

Volume corrente abaixo de $6 \mathrm{ml} / \mathrm{kg}$, com necessidade de reajuste maior que $20 \%$ da pressão inspiratórias (Pip) e pressão expiatória (PEEP) inicialmente programada

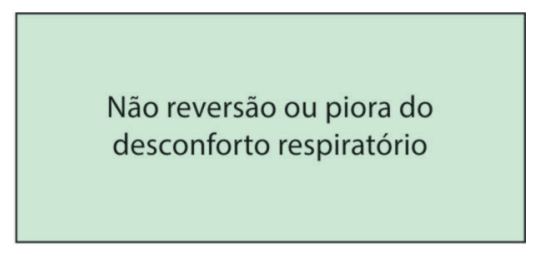

\section{VM- Parâmetros iniciais e estratégias ventilatórias}

As informações dos casos que necessitam de cuidados intensivos e uso de VM são muito escassos e limitados, de maneira geral, os dados são compatíveis com uma infecção viral respiratória semelhante a outras, como a influenza.

O consenso de especialistas chineses recomenda que, as crianças que forem submetidas a VNI por 2 horas sem melhora das condições ou não puderem tolerar VNI, com aumento das secreções das vias aéreas, tosse intensa ou instabilidade hemodinâmica, devem ser submetidas à ventilação mecânica invasiva imediatamente. A ventilação mecânica invasiva deve adotar "estratégia de ventilação de proteção pulmonar" de baixo volume corrente para reduzir a lesão pulmonar relacionada ao ventilador. Se necessário, pode ser aplicada ventilação em decúbito ventral, recrutamento pulmonar ou oxigenação por membrana extracorpórea $(\mathrm{ECMO})^{12}$. 
É importante reconhecer que, globalmente, existem poucas crianças internadas em unidades de terapia intensiva por COVID-19. Isso tem várias implicações: em primeiro lugar, há menos literatura publicada relacionada à prática pediátrica e, portanto, a orientação é inevitavelmente extrapolada da prática de consensos prévios na população infantil ou na adulta.

O Painel e membros convidados que fizeram parte do PEMVECC recomendam aderir em geral às recomendações sobre ventilação mecânica em crianças previamente publicadas e às recomendações práticas do PALICC nas questões específicas relacionadas à doença de COVID-19 em crianças $^{13,14}$.

Dada a falta de dados pediátricos, não é possível fazer recomendações específicas sobre o gerenciamento de casos pediátricos de COVID-19. O Painel recomenda a avaliação crítica de dados provenientes de adultos com COVID-19 e recomenda cuidado sobre o gerenciamento de COVID-19 antes de utilizá-los na prática clínica pediátrica diária.

Dados pediátricos preliminares mostram que a doença em sua forma grave parece infrequente em crianças pequenas, embora aqueles com $<1$ ano de idade possam ter formas mais graves da doença ${ }^{15}$.

\section{Na Prática}

O painel do PEMVECC recomenda que INTUBAÇÃO seja realizada por um especialista em vias aéreas em um ambiente fechado com uma quantidade mínima de funcionários. A videolaringoscopia (se disponível) deve ser usada. Todo o pessoal deve ter equipamento de proteção individual (EPI). O Painel recomenda pré-oxigenar o paciente com uma bolsa / máscara equipada com um filtro bacteriano / viral (Heat and moisture exchanger filter - HMEF). Se for necessária ventilação com bolsa / máscara, o Painel recomenda a "técnica de duas pessoas" para garantir uma melhor vedação da máscara ao redor da boca. Recomenda se sequência rápida de IOT, uso de tubos endotraqueais com cuff, inflando o manguito imediatamente após a intubação antes da verificação da posição do tubo por EtCO2, radiografia de tórax, ausculta ou exame de ultra-som ${ }^{16}$.

O painel recomenda ainda avaliar a complacência quase-estática (com respiração espontânea ausente) após a intubação sob condições de fluxo zero, sob ação do bloqueador neuromuscular, para registro e acompanhamento do baseline ${ }^{16}$.

\section{Configurações Ventilatórias Iniciais e Metas}

Os estudos até o momento não podem recomendar sobre o modo de ventilação que deve ser usado. Sugere-se seguir as diretrizes institucionais do seu serviço e o modo que a equipe está treinada para utilizar.

O Painel do PEMVECC recomenda a aplicação da estratégia ventilatória protetora de acordo com a recomendações atuais, com as estratégias recomendadas na ARDS em pediatria do PALICC ${ }^{17}$.

- volume corrente expirado (VCe) 5 - $7 \mathrm{~mL} / \mathrm{kg}$ de peso corporal ideal.

Atenção: podem ser necessários VCe menores (4 a $6 \mathrm{~mL} / \mathrm{kg}$ ) em condições de complacência do sistema respiratório mais baixas, ou seja, com doença pulmonar grave. Não há dados de casos pediátricos de COVID-19 sugerindo outras direções. A ideia é quanto mais grave, menor o pulmão, menor o VC que deve ser empregado. 

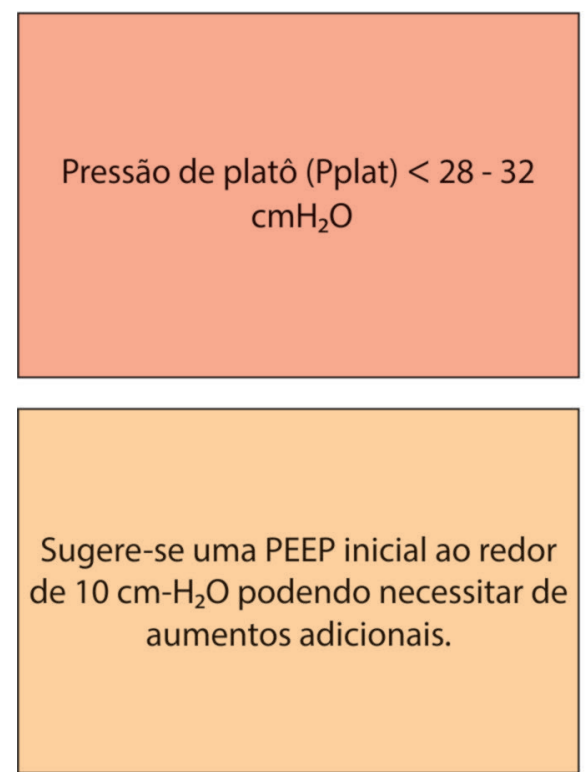

Driving pressure $\leq 15 \mathrm{cmH}_{2} \mathrm{O}$ seguindo as recomendações do PALICC
A frequência respiratória deve ser ajustada com base nos objetivos de ventilação minuto e no status ácido-

básico do paciente. Se necessário, pode ser mais elevada para compensar o volume minuto.

Existe orientação pediátrica limitada baseada em evidências onde há a sugestão de usar o guia/ tabela da ARDS Network da PEEP / $\mathrm{FiO}_{2}{ }^{18}$.

O painel do PEMVECC considera razoável titular a $\mathrm{FiO}_{2}$ para manter $\mathrm{SpO}_{2}$ entre 92 - 96\% na falta de quaisquer dados pediátricos específicos.

Para pacientes com doença grave o mínimo aceitável de valores de $\mathrm{SpO}_{2}$ deve ser $\mathbf{8 8 \%}$. O Painel recomenda ainda permitir hipercapnia permissiva, aceitando $\mathrm{pH}>7,20$, a menos que indicações clínicas específicas determinem o contrário ${ }^{18-20}$.

Sugere-se ainda considerar terapias escalonadas quando houver hipoxemia (refratária) definida por $\mathrm{PaO}_{2} / \mathrm{FiO}_{2}<150$; OI $\geq 12$; OSI $\geq 10$ e / ou $\mathrm{FiO}_{2}>0$,6. Pode-se então titular a PEEP, embora não se pode recomendar a melhor abordagem para titular a PEEP ou uma melhor maneira de realizar a manobra de recrutamento.

Os únicos dados pediátricos disponíveis (não incluindo pacientes com COVID-19) nas melhores programações de PEEP mostraram que não aderir à tabela da ARDSnet de baixa PEEP / $\mathrm{FiO}_{2}$ foi associado ao aumento da mortalidade em todas as causas por $\mathrm{pARDS}^{18}$, no entanto, o equilíbrio entre oxigenação e hemodinâmica permanece importante e não deve ser desconsiderado ao titular a PEEP.

Outra sugestão usada em pacientes adultos e que poderia ser utilizada em crianças é escolher uma PEEP mínima para prevenir o colapso gravitacional e ventilar da forma mais gentil possível buscando a menor pressão de distensão segura (Driving Presure). Assim, uma estratégia chamada de "mini titulação da PEEP" com o objetivo de encontrar uma PEEP mínima que diminua o colapso gravitacional e as hiperdistensão em regiões sem lesão pode ser utilizada. Se propõe com esta mini titulação uma manobra onde a PEEP poderá ser titulada de forma decremental iniciando de 18-16-14-12-10-8-6 $\mathrm{cmH}_{2} \mathrm{O}$ a cada dois minutos sem manobra de recrutamento alveolar prévia. A PEEP encontrada, em média tem sido de $10 \mathrm{cmH}_{2} \mathrm{O}$, embora possa variar muito entre os pacientes. A proposta é encontrar o valor de PEEP que resulta na menor driving pressure. Essa é uma estratégia que tem sido amplamente utilizada no mundo em protocolos de anestesia e parece ser a mais segura para o paciente COVID 1921. 


\section{Bloqueador Neuromuscular}

O Painel recomenda considerar o uso precoce de agentes bloqueadores neuromusculares (NMBA) por 24 a 48 horas na SDRAp moderada a grave (ou seja, $\mathrm{PaO}_{2} / \mathrm{FiO}_{2}<150$; OI $\geq 12$; OSI $\geq 10$ ). A justificativa para o uso do NMBA inclui evitar a respiração espontânea em altas pressões transpulmonares, minimizar a assincronia paciente/ventilador, necessidade de sedação profunda contínua, facilitar o posicionamento prono ou evitar ainda altas pressões de platô.

O Painel não pode recomendar qual limite de pressões de platô para iniciar o NMBA. Estes

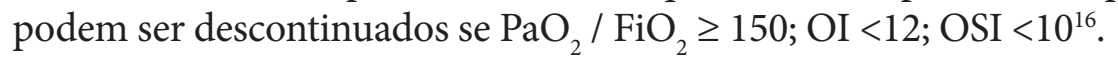

\section{Posição Prona}

O painel recomenda considerar o posicionamento em prono de forma precoce e prolongada na SDRAp moderada a grave (ou seja, $\mathrm{PaO}_{2} / \mathrm{FiO}_{2}<150$; OI $\geq 12$; OSI $\geq 10$ ).

\section{As práticas variam de 12 a 18 horas por dia com o paciente em decúbito ventral (posição prona)}

O posicionamento prono prolongado ( $>24$ horas) pode ser considerado no início da doença e já pode ser interrompido se $\mathrm{PaO}_{2} / \mathrm{FiO}_{2} \geq 150$; OI $<12$; OSI $<10^{16}$.

Em cenários com recursos limitados e poucos profissionais de saúde com materiais de proteção individual, a posição prona pode não ser possível, pois poderá aumentar desnecessariamente o risco de infecção e sobrecarga aos profissionais ${ }^{22}$.

\section{Oxido Nítrico Inalatório}

O PEMVECC sugeriu teste de óxido nítrico se for presumida alteração no reflexo de vasoconstrição pulmonar hipóxica, isto é, quando não houver melhora na oxigenação apesar de todas as outras medidas. Este pode ser especialmente o caso de pacientes com COVID-19 com complacência pulmonar normal ${ }^{16}$.

\section{Ventilação Oscilatória de Alta Frequência}

Achou-se razoável considerar a Ventilação Oscilatória de Alta Frequência - VOAF para hipoxemia refratária na SDRA induzida por COVID19 com complacência pulmonar e do sistema respiratório baixos usando uma titulação escalonada da pressão média das vias aéreas (PMVA). O momento da iniciação é influenciado por preferências pessoais, experiências institucionais, avaliação de riscos e disponibilidade de equipamentos. O Painel enfatiza que a VOAF deve ser considerada com cautela se houver pouca ou nenhuma experiência com esta modalidade.

Analisa-se ainda ser razoável considerar a Oxigenação por Membrana Extra-corpórea - ECMO se a hipoxemia refratária persistir, apesar de todas as medidas utilizadas. A disponibilidade limitada de recursos humanos e equipamentos pode influenciar a tomada de decisão. 


\section{Cuidados com a Criança em VM ${ }^{16}$}

- Todos os funcionários que entram na sala devem garantir que tenham EPI adequados

- Recomenda-se minimizar as desconexões do tudo endotraqueal (TET) e o uso do sistema fechado de aspiração (sucção fechada em linha). Não se pode fazer recomendações específicas sobre a umidificação das vias aéreas, mas deve-se dar preferência a filtros trocadores de umidade com filtros bacterianos / virais (HMEF) para a umidificação das vias aéreas, a fim de reduzir o risco de contaminação por aerossóis, também não há evidências fortes disso.

- A umidificação ativa pode levar o risco de contaminação por aerossol, enquanto a umidificação passiva requer alterações a cada 48 horas ou de acordo com a especificação de cada fabricante. Nesse momento deve-se o máximo gerir com atenção os recursos e dispositivos utilizados

- Recomenda-se o uso de filtros bacterianos / virais de barreira (HEPA) no ramo expiratório do circuito do paciente, com troca seguindo padrão da rotina hospitalar ou mais cedo, se eles se molharem para garantir eficiência total. O uso do HEPA é essencial quando a umidificação ativa for a de escolha

- O Painel recomenda a fixação imediata do tubo endotraqueal em caso de desconexão, seja esperada ou inesperada.

- O consenso do PEMVECC recomenda não realizar fisioterapia respiratória de rotina na ausência de muco espesso ou histórico de tampões nas vias aéreas e não usar dispositivos de auxílio à tosse.

- As estratégias ou bundles de prevenção da pneumonia associada a ventilação mecânica (PAV) devem ser asseguradas a fim de evitar o desenvolvimento de co-infecções que podem ocasionar aumento do tempo de VM e risco de morte ${ }^{22}$.

\section{Descontinuação e Extubação da VM}

O processo de descontinuação da ventilação mecânica pode ser lento ou rápido, dependo da condição clínica da criança. Ainda existem poucos dados publicados sobre a evolução clínica das crianças com a forma grave da COVID-19 ${ }^{15,23}$. Resultados iniciais de estudo de coorte em 46 UTIs norte americanas mostraram que entre 48 crianças diagnosticas com a COVID-19 apenas 18 (38\%) necessitaram de suporte ventilatório e a evolução clínica para a forma grave, com a necessidade de estratégias ventilatórias avançadas, menos comum que nos adultos, foi reportada em apenas 6 crianças ${ }^{24}$. Dessa forma, torna-se ainda mais difícil definir o momento exato em que o desmame deve ser iniciado.

Nesse contexto, recomenda-se seguir os critérios mais aceitos para iniciar o desmame ventilatório, que são $0^{25}$ :

- Condição clínica estável;

- Nutrição adequada;

- Drive ventilatório preservado;

- Parâmetros ventilatórios mínimos:

o $\mathrm{PEEP}<8 \mathrm{cmH}_{2} \mathrm{O}$;

o $\mathrm{FIO}_{2}<0,4-0,5$;

o Pressão de Pico $<30 \mathrm{cmH}_{2} \mathrm{O}$;

o FR programada $<15 \mathrm{rpm}$ para crianças menores ou 10 para crianças maiores/adolescentes. 
Os modos ventilatórios mais utilizados para o desmame em pediatria são PSV (com redução gradual da pressão de suporte) e CPAP (com redução gradual da PEEP) ${ }^{26}$. Todos esses modos podem ser utilizados em crianças com a COVID-19.

Recomenda-se que o Teste de Respiração Espontânea (TRE) seja realizado em CPAP de 5 $\mathrm{cmH}_{2} \mathrm{O}$ ou em PSV com PS de 8 a $10 \mathrm{cmH}_{2} \mathrm{O}$, por um período de 30 minutos a 2 horas. No entanto, é importante ressaltar que alguns estudos têm sugerido que o uso de PS acima da PEEP durante o TRE pode superestimar a capacidade da criança de manter a respiração espontânea após extubação ${ }^{27,28}$. Não se recomenda que o teste seja realizado utilizando um tubo T conectado a uma fonte oxigênio, pelo potencial de aumento da produção de aerossol e risco de contaminação da equipe multiprofissional.

Caso a criança mantenha estabilidade durante o TRE (volume corrente, frequência respiratória, padrão respiratório e $\mathrm{SpO}_{2}$ ), pode-se considerar a extubação. No entanto, apenas o TRE não é suficiente para detectar todas as crianças com risco de falha de extubação $0^{29}$.

Com o objetivo de reduzir a necessidade de aspiração nasotraqueal após extubação em pacientes com a COVID-19, considerando o risco de contaminação durante esse procedimento, sugere-se também a avaliação da patência e proteção de vias aéreas, por meio da eficácia da tosse (resposta ao estímulo pela sonda de aspiração), volume de secreção, frequência das aspirações traqueais, nível neurológico e teste de vazamento ("air leak" ou "cuffleak test")

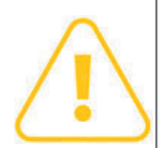

A extubação é uma intervenção que pode gerar grande quantidade de aerossóis, por isso é importante reforçar o correto uso dos EPI (avental, luvas, máscara N95 ou PFF2, óculos e/ou protetor facial). Além disso, recomenda-se que o fisioterapeuta se certifique que o número mínimo de profissionais necessários (geralmente dois) esteja presente no momento da extubação e, se possível, que seja realizada em quarto isolado, para reduzir o risco de contaminação ${ }^{31}$.

Deverão ser considerados todos os critérios de extubação segura ao paciente, para que possa ser avaliado qual a rota terapêutica ideal a ser definida previamente a extubação orotraqueal. A análise laboratorial e de exames de imagem, prévios a extubação, também auxiliam a determinar qual melhor estratégia ventilatória. A admissão na VNI após a extubação não deverá ser em caráter obrigatório a todos os pacientes, sendo avaliado cada caso clínico individualmente e traçado a melhor conduta de momento.

Após extubação, caso a criança apresente apenas $\mathrm{SpO}_{2}<94 \%$, sem sinais de desconforto respiratório, recomenda-se preferencialmente a utilização de cateter nasal de oxigênio até $6 \mathrm{~L} / \mathrm{min}$ (se $\mathrm{PaO}_{2}>75 \mathrm{mmHg}$ em ar ambiente) ou máscara com reservatório a $10 \mathrm{~L} / \mathrm{min}\left(\mathrm{se} \mathrm{PaO}_{2}\right.$ entre 63 e $75 \mathrm{mmHg}$ em ar ambiente) $)^{8}$. Se a criança apresentar $\mathrm{SpO}_{2}<94 \%$ e sinais de insuficiência respiratória ou $\mathrm{PaO}_{2}<$ $63 \mathrm{mmHg}$ (em ar ambiente), devem ser tomadas medidas preventivas para minimizar a aerossolização de dispositivos ou a tosse do paciente ${ }^{29}$.

A terapia inalatória na forma de nebulização após extubação (com adrenalina, por exemplo) também não é recomendada, pelo aumento da produção de aerossóis e risco de contaminação ${ }^{31,32}$. 
Na proposta de admissão da VNI após a extubação, considerar avaliação para período inicial prolongado ou intervalado, de acordo com quadro clínico do paciente em questão.

As sucessivas reavaliações após a extubação deste paciente deverão ser rigorosas e frequentes, nas primeiras 48 horas, devendo o fisioterapeuta estar alerta a qualquer sinal de falha de extubação (desconforto respiratório moderado/grave, $\mathrm{SpO}_{2}<94 \%$, rebaixamento do sensório, entrega total a frequência estabelecida na VNI mesmo em vigência da retirada de sedação, redução da entrada de ar e redução do volume corrente $<6 \mathrm{ml} / \mathrm{kg}$ ) sinalizando a equipe multidisciplinar quando o identificar.

\section{Conclusões}

Embora as evidências e experiências atuais mostrem que a SDRAp desencadeada por COVID-19 em crianças é de baixa prevalência, o comportamento em nosso país ainda não é estimado. Na vigência de insuficiência respiratória deve-se iniciar a intervenção com ventilação não invasiva, sem que haja prolongamento do seu tempo de uso, seguida para ventilação mecânica invasiva de maneira precoce. Parâmetros ventilatórios gentis e protetores devem ser usados para evitar mais lesão pulmonar. $\mathrm{O}$ quarto de isolamento, ou aspiração negativa preferencialmente, associado ao uso de EPI pela equipe são requisitos primordiais para minimizar transmissão do vírus SAR-Cov2. Desta forma, precisamos estar atentos à presença de agentes virais habituais durante o período de outono/inverno. Por esse motivo, este documento baseia-se em aspectos técnicos de estudos recentes e em andamento, relacionados ao uso da ventilação mecânica no contexto do paciente pediátrico com suspeita ou confirmação de COVID-19.

\section{Referências}

1. Brasil. Ministério da Saúde. Brasil registra 19.638 casos confirmados de coronavírus e 1.056 mortes [Internet]. Brasília: Ministério da Saúde; 2020 [cited 2020 Apr 11]. Available from: https://www.saude. gov.br/noticias/agencia-saude/46709-brasil-registra-19-638-casos-confirmados-de-coronavirus-e-1$\underline{057-m o r t e s}$

2. Centers for Disease Control and Prevention. Coronavirus disease 2019 (COVID-19): situation summary [Internet]. Atlanta: Centers for Disease Control and Prevention; 2020. Available from: https:// www.cdc.gov/ coronavirus/2019-ncov/summary.html

3. Kelvin AA, Halperin S. COVID-19 in children: the link in the transmission chain. Lancet Infect Dis. 2020 Jun;20(6):633-634. doi: 10.1016/S1473-3099(20)30236-X. Epub 2020 Mar 25.

4. Chen ZM, Fu JF, Shu Q. New coronavirus: new challenges for pediatricians. World J Pediatr. 2020 Jun;16(3):222. doi: 10.1007/s12519-020-00346-4. Epub 2020 Feb 10.

5. Chen ZM, Fu JF, Shu Q, Chen YH, Hua CZ, Li FB, et al. Diagnosis and treatment recommendations for pediatric respiratory infection caused by the 2019 novel coronavirus. World J Pediatr. 2020 Jun;16(3):240-246. doi: 10.1007/s12519-020-00345-5. Epub 2020 Feb 5.

6. Qiu H, Wu J, Liang H, Yunling L, Song Q, Chen D. Clinical and epidemiological features of 36 children with coronavirus disease 2019 (COVID-19) in Zhejiang, China: an observational cohort study. Lancet Infect Dis. 2020 Jun;20(6):689-696. doi: 10.1016/S1473-3099(20)30198-5. Epub 2020 Mar 25.

7. Kneyber MCJ, Medina A, Modesto i Alapont V, Blokpoe R, Brierley J, Chidini G, et al. Practice recommendations for managing children with proven or suspected COVID-19 [Internet]. Geneva: European Society for Paediatric and Neonatal Intensive Care; 2020. Available from: https://espnic- 
online.org/News/Latest-News/Practice-recommendations-for-managing-children-with-proven-orsuspected-COVID-19

8. ASSOBRAFIR. Aspectos epidemiológicos e atuação do fisioterapeuta na prevenção e tratamento da covid-19 na população infantil em ambiente hospitalar. Comunicação Oficial ASSOBRAFIR [Internet]. Brasília: Associação Brasileira de Fisioterapia Cardiorrespiratória e Fisioterapia em Terapia Intensiva; 2020. Available from: https://assobrafir.com.br/covid-19 pediatria/

9. Ferrés J. Comparison of two nebulized treatments in wheezing infants. Eur Respir J 1988; 1 (Suppl):306.

10. García-Sosa A, Orozco-Romero DP, Iglesias-Leboreiro J, Bernárdez-Zapata I, Rendón-Macías ME. Escala Wood Downes-Ferrés, una opción útil para identificar la gravedad en crisis asmática. Rev Mex Pediatr. 2018 Ene-Feb;85(1):11-16.

11. Majumder MS, Mandl KD. Early transmissibility assessment of a novel coronavirus in Wuhan, China. SSRN 2020; published online Jan 23 (version 1). SSRN. 2020 Jan 24 [cited 2020 Mar 20]:3524675. doi: 10.2139/ssrn.3524675. Preprint.

12. Shen K, Yang Y, Wang T, Zhao D, Jiang Y, Jin R et al. Diagnosis, treatment and prevention of 2019 novel coronavirus infection in children: expert's consensus statement. World J Pediatr. 2020 Jun;16(3):223231. doi: 10.1007/s12519-020-00343-7. Epub 2020 Feb 7.

13. Kneyber MCJ, de Luca D, Calderini E, Jarreau PH, Javouhey E, Lopez-Herce J, et al. Recommendations for mechanical ventilation of critically ill children from the Paediatric Mechanical. Intensive Care Med. 2017 Dec;43(12):1764-1780. doi: 10.1007/s00134-017-4920-z. Epub 2017 Sep 22.

14. Kneyber MCJ, de Luca D, Calderini E, Jarreau P-H, Javouhey E, Lopez-Herce J, et al. Recommendations for mechanical ventilation of critically ill children from the Paediatric Mechanical Ventilation Consensus Conference (PEMVECC). Intensive Care Med. 2017 Dec;43(12):1764-1780. doi: 10.1007/s00134-017-4920-z. Epub 2017 Sep 22. Pediatric Acute Lung Injury Consensus Conference Group. Pediatric acute respiratory distress syndrome: consensus recommendations from the Pediatric Acute Lung Injury Consensus Conference. Pediatr Crit Care Med. 2015 Jun;16(5):428-39. doi: 10.1097/ PCC.0000000000000350.

15. Dong Y, Mo X, Hu Y, Qi X, Jiang F, Jiang Z, et al. Epidemiological Characteristics of 2143 Pediatric Patients With 2019 Coronavirus Disease in China. Pediatrics. e20200702. doi: 10.1542/peds.2020-0702.

16. Kneyber MCJ, Medina A, Modesto i Alapont V, Blokpoe R, Brierley J, Chidini G, et al. Practice recommendations for the management of children with suspected or proven COVID-19 infections from the Paediatric Mechanical Ventilation Consensus Conference (PEMVECC) and the section Respiratory Failure from the European Society for Paediatric and Neonatal Intensive Care (ESPNIC) [Internet]. Geneva: European Society for Paediatric and Neonatal Intensive Care; 2020. Available from: https:/espnic-online.org/content/download/3951/20876/file/2020 ESPNIC PEMVECC COVID-19 practice recommendations.pdf

17. Calvo C, García López-Hortelano M, de Carlos Vicente JC, Vázquez Martínez JL. Recomendaciones sobre el manejo clínico de la infección por el «nuevo coronavirus» SARS-CoV2. Grupo de trabajo de la Asociación Espanhola de Pediatría (AEP). An Pediatr (Barc) ; 92(4): 241.e1-241.e11, 2020 Apr.

18. Khemani RG, Parvathaneni K, Yehya N, Bhalla AK, Thomas NJ, Newth CJL. PEEP Lower Than the ARDS Net- work Protocol is Associated with Higher Pediatric ARDS Mortality. Am J Respir Crit Care 
Med. 2018 Jul 1;198(1):77-89. doi: 10.1164/rccm.201707-1404OC.

19. Schmidt B, Whyte, RK. Oxygen saturation target ranges and alarm settings in the NICU: What have we learnt from the neonatal oxygenation prospective meta-analysis (NeOProM)? Semin Fetal Neonatal Med. 2020 Apr;25(2):101080. doi: 10.1016/j.siny.2020.101080. Epub 2020 Jan 16.

20. Martin D, Peters, MJ. Might children rust? What are the risks of supplemental oxygen in acute illness. Arch Dis Child. 2019 Feb;104(2):106-107. doi: 10.1136/archdischild-2018-315437. Epub 2018 Sep 13.

21. Barbas CS, Ísola AM, Farias AM, Cavalcanti AB, Gama AM, Duarte AC, et al. Recomendações Brasileiras de Ventilação Mecânica 2013. Parte I. Rev Bras Ter Intensiva. 2014 Jun;26(2):89-121. doi: 10.5935/0103-507X.20140017

22. Sundaram M, Ravikumar N, Bansal A, Nallasamy K, Basavaraja GV, Rakesh L, et al. Novel Coronavirus 2019 (2019-nCoV) Infection: Part II- Respiratory support in the Pediatric Intensive Care Unit in resource-limited settings. Indian Pediatr. 2020 Apr 15;57(4):335-342. doi: 10.1007/s13312-0201786-x. Epub 2020 Mar 29.

23. Cai J, Xu J, Lin D, Yang Z, Xu L, Qu Z, Zhang Y, et al. A Case Series of children with 2019 novel coronavirus infection: clinical and epidemiological features. Clin Infect Dis. 2020 Feb 28:ciaa198. doi: 10.1093/cid/ciaa198. Online ahead of print.

24. Shekerdemian LS, Mahmood NR, Wolfe KK, Riggs BJ, Ross CE, McKiernan CA, et al.: Characteristics and Outcomes of Children With Coronavirus Disease 2019 (COVID-19) Infection Admitted to US and Canadian Pediatric Intensive Care Units. JAMA Pediatr. 2020 May 11. doi: 10.1001/ jamapediatrics.2020.1948. Online ahead of print.

25. Walsh BK. Neonatal and Pediatric Respiratory Care. $4^{\text {th }}$ ed. St Louis: Elsevier; 2015. 331-333 p.

26. Newth CJL, Venkataraman S, Willson DF, Meert KL, Harrison R, Dean JM, et al. Weaning and extubation readiness in pediatric patients. Pediatr Crit Care Med. 2009 Jan;10(1):1-11. doi: 10.1097/ PCC.0b013e318193724d.

27. Ferguson LP, Walsh BK, Munhall D, Arnold JH. A spontaneous breathing trial with pressure support overestimates readiness for extubation in children. Pediatr Crit Care Med. 2011 Nov;12(6):e330-5. doi: 10.1097/PCC.0b013e3182231220.

28. Khemani RG, Hotz J, Morzov R, Flink RC, Kamerkar A, LaFortune M, et al. Pediatric extubation readiness tests should not use pressure support. Intensive Care Med. 2016 Aug;42(8):1214-22. doi: 10.1007/s00134-016-4387-3. Epub 2016 Jun 18.

29. Quintard H, L'Her E, Pottecher J, Adnet F, Constantin J-M, De Jong A, et al. Experts' guidelines of intubation and extubation of the ICU patient of French Society of Anaesthesia and Intensive Care Medicine (SFAR) and French-speaking Intensive Care Society (SRLF). Ann Intensive Care. 2019 Jan 22;9(1):13. doi: 10.1186/s13613-019-0483-1.

30. Wratney AT, Cheifetz IM. Extubation criteria in infants and children. Respir Care Clin N Am. 2006 Sep;12(3):469-81. doi: 10.1016/j.rcc.2006.05.005.

31. Lazzeri M, Lanza A, Bellini R, Bellofiore A, Cecchetto S, Colombo A, et al. Respiratory physiotherapy in patients with COVID-19 infection in acute setting: a Position Paper of the Italian Association of 
Respiratory Physiotherapists (ARIR). Monaldi Arch Chest Dis. 2020 Mar 26;90(1). doi: 10.4081/ monaldi.2020.1285.

32. O'Neil CA, Li J, Leavey A, Wang Y, Hink M, Wallace M, et al. Characterization of Aerosols Generated During Patient Care Activities. Clin Infect Dis. 2017 Oct 15;65(8):1335-1341. doi: 10.1093/cid/cix535.

Submissão em: 08/06/2020

Aceite em: 15/07/2020 